\title{
Desempenho de larvas de Helicoverpa armigera (Hübner) (Lepidoptera: Noctuidae) em diferentes fontes alimentares ${ }^{1}$
}

\author{
Crislaine Sartori Suzana², Raquel Damiani², Letícia Slongo Fortuna ${ }^{2}$, José Roberto Salvadori²
}

\begin{abstract}
Performance of Helicoverpa armigera (Hübner) (Lepidoptera: Noctuidae) larvae in different food sources

Recently observed in Brazil, Helicoverpa armigera became a relevant pest due to its rapid spread and the economic importance of crops in which it has established, such as soybean and maize. Understanding its establishment process in different regions and production systems, as well as the population dynamics of a polyphagous pest, as the basis for its management, depends on the knowledge of the effect of plant species as food sources on the pest biology. A laboratory experiment was conducted, supplying the caterpillars with reproductive organs of soybean, maize, canola, black oat, oat, turnip and ryegrass. It was concluded that the different food sources affect the larval development of $H$. armigera. Maize and wheat ears and canola siliques are the best food sources for the development of H. armigera. Ryegrass ears, on the other hand, are the worst ones. Black oat and oat panicles and turnip siliques are less suitable than soybean pods as food sources for the caterpillars.
\end{abstract}

KEY-WORDS: Insect pest; host plants; biological parameters.

\section{INTRODUÇÃO}

Helicoverpa armigera (Lepidoptera: Noctuidae: Heliothinae) é considerada uma das pragas agrícolas mais importantes no mundo, devido à sua ampla distribuição geográfica e comportamento polífago (Zalucki et al. 1986, Guo 1997, Behere et al. 2013). Recentemente, foi identificada no continente americano, primeiro no Brasil (Czepak et al. 2013b) e, em seguida, no Paraguai (Paraguay 2013) e na Argentina (Murúa et al. 2014).

Sua ocorrência já foi registrada em mais de 200 espécies de plantas hospedeiras, cultivadas e silvestres, pertencentes a aproximadamente 67 famílias, incluindo Asteraceae, Fabaceae, Malvaceae, Poaceae e Solanaceae (Reed \& Powar 1982,

\section{RESUMO}

Recentemente observada no Brasil, Helicoverpa armigera tornou-se praga relevante, tanto pela rápida disseminação como pela importância econômica das culturas nas quais se estabeleceu, como a soja e o milho. A compreensão do processo de estabelecimento em diferentes regiões e sistemas de produção e da dinâmica populacional de uma praga reconhecidamente polífaga, como base para o seu manejo, depende do conhecimento sobre o efeito de espécies vegetais como alimento, na biologia da espécie. Conduziu-se experimento em laboratório, fornecendose às lagartas órgãos reprodutivos de soja, milho, canola, aveia-preta, aveia-branca, nabo e azevém. Concluiu-se que as diferentes fontes alimentares afetam o desenvolvimento larval de $H$. armigera. Espigas de milho e de trigo e síliquas de canola são os melhores alimentos para o desenvolvimento de H. armigera. Espigas de azevém, por outro lado, são o pior alimento. Panículas de aveia-preta e aveia-branca e síliquas de nabo são menos adequadas que vagens de soja, como fonte de alimento para as lagartas.

PALAVRAS-CHAVE: Inseto-praga; plantas hospedeiras; parâmetros biológicos.

Pogue 2004, Sharma et al. 2005, Ali \& Choudhury 2009).

No Brasil, $H$. armigera encontra-se amplamente disseminada e é considerada praga em várias culturas, especialmente em soja e algodão, no Cerrado (Ávila et al. 2013, Czepak et al. 2013a, Specht et al. 2013). No Sul do País, está estabelecida como praga de soja, mas também ocorre em espigas de milho e, na entressafra da soja, tem sido encontrada em espigas de trigo, síliquas de canola e cápsulas de linho, bem como em espécies de plantas espontâneas, como nabo e aveia-preta (Guedes et al. 2013, Arnemann et al. 2014, Salvadori \& Suzana 2014).

A polifagia é um recurso para a sobrevivência de fitófagos que assume grande importância, com relação ao desempenho biológico e à dinâmica 
populacional de pragas. Em uma mesma região, espécies polífagas podem ser pragas de uma ou mais culturas, ou apenas se manterem em baixa densidade em diferentes plantas hospedeiras, até encontrarem um alimento capaz de sustentar o seu completo desenvolvimento (Moscardi et al. 2012).

As lagartas de $H$. armigera se alimentam de folhas, especialmente brotos, bem como inflorescências, frutos e vagens (Reed 1965, Moral-Garcia 2006, Sullivan \& Molet 2014). Danos podem decorrer de ataques nas fases vegetativa e reprodutiva das culturas (Czepak et al. 2013b), levando a perdas econômicas elevadas (Fitt 1989, Czepak et al. 2013b, Tay et al. 2013). No entanto, a quantidade e a qualidade do alimento consumido, bem como a eficiência como este é utilizado, é que determinam as condições nutricionais para o crescimento e proliferação de insetos (Ruan \& Wu 2001, Barton \& Raubenheimer 2003).

O conhecimento sobre as exigências nutricionais de uma praga pode auxiliar na compreensão da sua dinâmica populacional, na definição do potencial de danos e nas decisões para controle (Kogan \& Parra 1981, Slanky Junior \& Wheeler 1987, Parra 1991, Cohen 2004, Jervis 2005), principalmente em sistemas de produção diversificados, como os existentes no Brasil.

Diante do exposto, objetivou-se avaliar o desempenho de larvas de $H$. armigera alimentadas em órgãos reprodutivos de espécies vegetais cultivadas e espontâneas, associadas ao sistema de produção de soja, na região Sul do Brasil.

O trabalho foi realizado em casa-de-vegetação e câmara climatizada $\left(25 \pm 0,5{ }^{\circ} \mathrm{C}\right.$, umidade relativa do ar de $60 \pm 10 \%$ e fotofase de 12 horas), na Faculdade de Agronomia e Medicina Veterinária da Universidade de Passo Fundo, em Passo Fundo (RS). Utilizaram-se lagartas de terceira geração, criadas com dieta artificial (Greene et al. 1976), a partir de lagartas coletadas em lavoura de soja local, em janeiro de 2014.

As dietas vegetais fornecidas como fonte de alimento às lagartas foram: vagem de soja (Glycine max), espiga de trigo (Triticum aestivum), panícula de aveia-preta (Avena strigosa), panícula de aveia-branca (Avena sativa), espiga de milho sem palha (Zea mays), síliqua de canola (Brassica napus), síliqua de nabo (Raphanus sativus) e espiga de azevém (Lolium multiflorum). Na escolha das espécies, consideraram-se plantas hospedeiras reais, com base em constatações de campo (Salvadori et al. 2013,
Salvadori \& Suzana 2014), e outras potenciais (presentes em campo, na entressafra da cultura da soja).

As plantas foram cultivadas em vasos, em casa-de-vegetação, de onde foram coletados os órgãos reprodutivos para alimentar as lagartas. A coleta do material foi feita quando as sementes encontravam-se verdes, duras e cheias em soja; leitosas em milho e em azevém; e pastosas nas demais espécies.

Foram individualizadas 50 larvas no início do $4^{\circ}$ instar, para cada tipo de alimento, exceto milho, em que foram utilizadas 32 (número disponível de lagartas da idade e do tamanho padronizados). O uso de lagartas de $4^{\circ}$ instar baseou-se no comportamento de $H$. armigera, que, em condições naturais, alimenta-se de partes vegetativas nos primeiros instares, para depois explorar os órgãos reprodutivos de seus hospedeiros (Farrar \& Bradley 1985, Rogers \& Brier 2010, Bortolotto et al. 2014).

O desempenho das larvas de H. armigera nos diferentes alimentos foi avaliado quanto aos seguintes parâmetros biológicos: sobrevivência, duração e peso (ao $7^{\circ}$ dia após a infestação) de larvas e peso de pupas (com 24 horas).

Os dados foram submetidos à análise de variância e as médias comparadas pelo teste Tukey $(\mathrm{p} \leq 0,05)$, em delineamento inteiramente casualizado, com oito tratamentos (espécies vegetais) e número variável de repetições (em função da mortalidade). Para a análise da sobrevivência, as lagartas foram reunidas em cinco repetições de dez indivíduos.

O consumo das lagartas concentrou-se nas sementes, depois de essas terem sido acessadas por meio da perfuração dos tecidos que envolvem os órgãos reprodutivos oferecidos como alimento. Mesmo já tendo atingido as sementes, as lagartas também comeram, mas em menor quantidade, porções do envoltório externo das sementes de soja, canola, nabo, azevém, trigo, aveias e milho.

A sobrevivência das lagartas foi afetada pelo alimento ingerido, atingindo maior valor em milho e canola, sem diferir do que foi observado em soja (Tabela 1). Embora não tenha diferido da soja, a sobrevivência das lagartas em nabo, trigo e aveia-preta foi inferior à encontrada em milho e em canola. Em aveia-branca, a sobrevivência das lagartas foi baixa, sendo inferior à observada em soja. Em azevém, nenhuma das lagartas sobreviveu.

O tempo necessário para completar o desenvolvimento larval foi mais curto em milho, seguido da soja (Tabela 1). Nas aveias e no nabo, o desen- 
volvimento das lagartas foi mais demorado, em comparação aos demais alimentos, superando o tempo observado em soja em 50,9 \% com aveia-branca, $66,7 \%$ com aveia-preta e $76,3 \%$ com nabo.

A taxa de sobrevivência e a velocidade para completar o desenvolvimento na fase larval (Tabelas 1 e 2) indicam que, além da soja, $H$. armigera tem seus requisitos alimentares supridos também pelo milho e pela canola. Esses resultados são coerentes com as elevadas taxas de sobrevivência já registradas em milho (Jallow \& Zalucki 1996, Liu et al. 2004, Amer \& El-Sayed 2014) e justificam a ocorrência de lagartas bem desenvolvidas, ou seja, no último instar, em lavouras de canola, próximo à colheita (Salvadori \& Suzana 2014). Por outro lado, mostram que, mesmo ingerido sem restrição, o azevém não constitui alimento adequado para lagartas de $H$. armigera

Tabela 1. Sobrevivência e duração de larvas de H. armigera alimentadas com diferentes fontes alimentares, em laboratório, sob $25 \pm 0,5^{\circ} \mathrm{C}, 60 \pm 10 \%$ UR e 12 horas de fotofase (Passo Fundo, RS, 2014).

\begin{tabular}{lccc}
\hline \multicolumn{1}{c}{ Alimento } & $\begin{array}{c}\text { Sobrevivência }{ }^{1} \\
(\%)\end{array}$ & $\begin{array}{c}\text { Duração } \\
(\text { dias })\end{array}$ & $\mathrm{n}$ \\
\hline Espiga de milho & $94,0 \pm 2,4 \mathrm{a}$ & $8,2 \pm 0,2 \mathrm{a}$ & 30 \\
Síliqua de canola & $94,0 \pm 4,0 \mathrm{a}$ & $13,9 \pm 0,2 \mathrm{c}$ & 47 \\
Vagem de soja & $72,0 \pm 7,3 \mathrm{ab}$ & $11,5 \pm 0,4 \mathrm{~b}$ & 36 \\
Síliqua de nabo & $64,0 \pm 6,7 \mathrm{~b}$ & $20,1 \pm 0,5 \mathrm{e}$ & 32 \\
Espiga de trigo & $70,0 \pm 3,1 \mathrm{~b}$ & $14,3 \pm 0,3 \mathrm{c}$ & 34 \\
Panícula de aveia-preta & $52,0 \pm 4,9 \mathrm{bc}$ & $19,0 \pm 0,5 \mathrm{e}$ & 26 \\
Panícula de aveia-branca & $38,0 \pm 6,6 \mathrm{c}$ & $16,8 \pm 0,8 \mathrm{~d}$ & 19 \\
Espiga de azevém & $0,0 \pm 0,0 \mathrm{~d}$ & - & - \\
\hline C.V. (\%) & 18,5 & 16,2 & \\
\hline
\end{tabular}

Médias \pm EP seguidas pela mesma letra não diferem entre si pelo teste Tukey ( $p \leq 0,05) ;{ }^{1}$ número inicial: 50 lagartas, exceto em milho (32 lagartas).

Tabela 2. Peso de larvas e pupas de $H$. armigera alimentadas com diferentes fontes alimentares, em laboratório, sob $25 \pm 0,5^{\circ} \mathrm{C}, 60 \pm 10 \%$ UR e 12 horas de fotofase (Passo Fundo, RS, 2014).

\begin{tabular}{lcccc}
\hline Alimento & Larva $(\mathrm{mg})$ & $\mathrm{n}$ & Pupa $\left.^{(\mathrm{mg})}\right)^{1}$ & $\mathrm{n}$ \\
\hline Vagem de soja & $319,9 \pm 11,8 \mathrm{a}$ & 24 & $285,6 \pm 7,9 \mathrm{a}$ & 36 \\
Síliqua de canola & $311,8 \pm 12,3 \mathrm{a}$ & 49 & $274,6 \pm 5,3 \mathrm{a}$ & 47 \\
Espiga de milho & $258,5 \pm 7,2 \mathrm{~b}$ & 20 & $238,7 \pm 4,9 \mathrm{~b}$ & 30 \\
Espiga de trigo & $258,0 \pm 15,4 \mathrm{~b}$ & 37 & $237,3 \pm 5,9 \mathrm{~b}$ & 34 \\
Panícula de aveia-branca & $135,0 \pm 11,8 \mathrm{c}$ & 20 & $159,8 \pm 5,9 \mathrm{c}$ & 19 \\
Síliqua de nabo & $129,3 \pm 6,1 \mathrm{c}$ & 42 & $161,7 \pm 4,5 \mathrm{c}$ & 32 \\
Panícula de aveia-preta & $103,0 \pm 4,9 \mathrm{c}$ & 33 & $141,7 \pm 6,9 \mathrm{c}$ & 26 \\
Espiga de azevém & $92,8 \pm 7,0 \mathrm{c}$ & 14 & - & - \\
\hline C.V. (\%) & 30,0 & \multicolumn{4}{c}{15,1} & \\
\hline
\end{tabular}

Médias \pm EP seguidas pela mesma letra não diferem entre si pelo teste Tukey $(\mathrm{p} \leq 0,05)$.
Aveia-preta, aveia-branca e nabo, além de proporcionarem baixa viabilidade larval, determinaram o prolongamento da fase larval, o que evidencia uma situação de deficiência alimentar (Parra \& Haddad 1989, Hwang et al. 2008, Kouhi et al. 2014).

$\mathrm{O}$ crescimento dos insetos, avaliado pelo peso das lagartas e das pupas, variou com os alimentos fornecidos (Tabela 2). Para ambas as fases, o melhor desempenho foi constatado em soja e canola, seguidas de milho e trigo. Os menores pesos foram observados em aveia-branca, aveia-preta, nabo e azevém, sendo que, neste último, nenhum indivíduo atingiu a fase de pupa.

Maior taxa de crescimento em lagartas (Hwang et al. 2008) e maior peso de larvas e de pupas de H. armigera (Liu et al. 2004, 2006) estão associados à maior adequação do alimento ingerido, o que, normalmente, reflete em melhor desempenho reprodutivo dos adultos (Daryaei et al. 2007, Parra 2009).

O baixo peso de larvas alimentadas com aveia-preta, aveia-branca, nabo e azevém permite inferir que o prolongamento do período larval (Tabela 1) é resultado da má qualidade do alimento, que não permite adequada nutrição para o desenvolvimento do inseto. Além disso, as pupas geradas com esses alimentos também apresentaram peso menor que nos demais (Tabela 2).

A adequação de uma planta como hospedeira depende da sua composição química (Bernys \& Chapman 1994), incluindo nutrientes e substâncias secundárias (aleloquímicos), bem como da sua capacidade de digestão e assimilação pelo inseto (Martin \& Pulin 2004, Naseri \& Gatehouse 2010, Namin et al. 2014).

No presente estudo, em todas as espécies vegetais, as lagartas consumiram avidamente e defecaram dentro de padrões normais, quanto ao aspecto e à quantidade, nada ocorrendo que pudesse sugerir alguma restrição ao consumo. Dessa forma, o mau desempenho biológico de $H$. armigera, em algumas das plantas oferecidas, pode ser atribuído a aspectos nutricionais das plantas avaliadas (Parra 2009).

Considerando-se os sistemas de sucessão e de rotação de culturas empregados na Região Sul do Brasil, os resultados obtidos para o desempenho biológico na fase larval de $H$. armigera, em diferentes espécies vegetais, indicam que existem "pontes verdes" para sustentar as populações da praga na maior parte do ano, desde que não haja limitações 
climáticas, especialmente baixas temperaturas, como as que ocorrem no inverno.

Em regiões onde os cultivos de trigo e de canola antecedem o de soja, pelo menos uma parte das infestações de $H$. armigera que ocorrem já no início do desenvolvimento da soja pode ser oriunda de mariposas, cujas larvas completaram o ciclo naquelas lavouras de inverno-primavera. Em aveia-preta, aveia-branca e nabo, a taxa de pupação é menor, o que diminui a probabilidade de que nessas espécies vegetais se originem populações de $H$. armigera que venham a infestar a soja recém-emergida, cultivada em sucessão. Já o azevém poderia servir para manter lagartas no sistema apenas temporariamente, uma vez que elas não sobrevivem ao se alimentarem de suas espigas.

Estudos complementares, que avaliem a ingestão e o aproveitamento do alimento na fase larval e o efeito do alimento nos parâmetros biológicos da fase adulta, são necessários para uma melhor compreensão da importância de espécies vegetais na biologia e na dinâmica populacional de $H$. armigera, na condição de praga recém-introduzida no Brasil.

Concluiu-se que as diferentes fontes alimentares afetam o desenvolvimento larval de $H$. armigera. Espigas de milho e trigo, síliquas de canola e vagens de soja são os melhores alimentos para o desenvolvimento da $H$. armigera. Por outro lado, espigas de azevém são o pior alimento para a nutrição e desenvolvimento da praga. Panículas de aveia-preta e de aveia-branca e síliquas de nabo são menos adequadas que vagens de soja, como alimento para as lagartas.

\section{REFERÊNCIAS}

ALI, A.; CHOUDHURY, R. A. Some biological characteristics of Helicoverpa armigera on chickpea. Tunisian Journal of Plant Protection, Kef, v. 4, n. 1, p. 99-106, 2009.

AMER, A. E. A.; EL-SAYED, A. A. A. Effect of different host plants and artificial diet on Helicoverpa armigera (Hubner) (Lepidoptera: Noctuidae) development and growth index. Jornal of Entomology, New York, v. 11, n. 5, p. 299- 205, 2014.

ARNEMANN, J. A. et al. Até no inverno. Cultivar Grandes Culturas, Pelotas, n. 182, p. 26-28, 2014.

ÁVILA, C. J.; VIVAN, L. M.; TOMQUELSKI, G. V. Ocorrência, aspectos biológicos, danos e estratégias de manejo de Helicoverpa armigera (Hübner) (Lepidoptera:
Noctuidae) nos sistemas de produção agrícolas. Dourados: Embrapa, 2013. (Circular técnica, 23).

BARTON, L. B.; RAUBENHEIMER, D. Ontogenetic changes in the rate of ingestion and estimates of food consumption in fourth and fifth instar Helicoverpa armigera caterpillars. Journal of Insect Physiology, Oxford, v. 49, n. 1, p. 63-71, 2003.

BEHERE, G. T. et al. Population genetic structure of the cotton bollworm Helicoverpa armigera (Hubner) (Lepidoptera: Noctuidae) in India as inferred from EPICPCR DNA Markers. Plos One, San Francisco, v. 8, n. 1, p. 53448, 2013.

BERNYS, E. A.; CHAPMAN, R. F. Host-plant selection by phytophagous insects. New York: Chapman \& Hall, 1994.

BORTOLOTTO, O. C. et al. Características biológicas de Heliothis virescens alimentados com Bt-soybean MON $87701 \times$ MON 89788 e sua isolinha convencional. Anais da Academia Brasileira de Ciências, Rio de Janeiro, v. 86, n. 2, p. 973-980, 2014.

COHEN, A. C. Insect diet: science and technology. Boca Raton: CRC, 2004.

CZEPAK, C. et al. Como manejas: táticas racionais, sustentáveis e integradas, adotadas em conjunto e de forma ampla são o caminho correto do complexo de lagartas Heliothinae e o enfrentamento dos ataques sucessivos de Helicoverpa armigera em lavouras de soja, milho e algodão. Cultivar Grandes Culturas, Pelotas, n. 171, p. 6-10, 2013a.

CZEPAK, C. et al. Primeiro registro de ocorrência de Helicoverpa armigera (Hübner) (Lepidoptera: Noctuidae) no Brasil. Pesquisa Agropecuária Tropical, Goiânia, v. 43, n. 1, p. 110-113, 2013b.

DARYAEI, G. M. et al. Host preference and nutrition efficiency of the gypsy moth, Lymantria dispar L. (Lymantriidae: Lepidoptera), on different poplar clones. Journal of Agricultural and Food Chemistry, Washington, DC, v. 32, n. 6, p. 469-476, 2007.

FARRAR, R. R.; BRADLEY, J. R. Within-plant distribution of Heliothis spp. (Lepidoptera: Noctuidae) eggs and larvae on cotton in North Carolina. Environmental Entomology, Oxford, v. 14, n. 3, p. 205-209, 1985.

FITT, G. P. The ecology of Heliothis species in relation to agroecosystems. Annual Review of Entomology, Palo Alto, v. 34, n. 1, p. 17-52, 1989.

GREENE, G. L.; LEPPLA, N. C.; DICKERSON, W. A. Velvetbean caterpillar: a rearing procedure and artificial diet. Journal of Economic Entomology, Lanham, v. 69, n. 4, p. 487-488, 1976. 
GUEDES, J. C. V. et al. Manejar ou perder. Cultivar Grandes Culturas, Pelotas, n. 176, p. 12-16, 2013.

GUO, Y. Y. Progress in the research on migration regularity of Helicoverpa armigera and relationships between the pest and its host plants. Acta Entomologia Sinica, Beijing, v. 40, n. 1, p. 1-6, 1997.

HWANG, S. Y.; LIU, C. H.; SHEN, T. C. Effects of plant nutrient availability and host plant species on the performance of two Pieris butterflies (Lepidoptera: Pieridae). Biochemical Systematics and Ecology, New York, v. 36, n. 7, p. 505-513, 2008.

JALLOW, M. F. A.; ZALUCKI, M. P. Within- and between- population variation in host-plant preference and specificity in Australian Helicoverpa armigera (Hübner) (Lepidoptera: Noctuidae). Australian Journal of Zoology, Osaka, v. 44, n. 5, p. 503-519, 1996.

JERVIS, M. A. Insect as natural enemies: a practical perspective. New York: Springer, 2005.

KOGAN, M.; PARRA, J. R. P. Techniques and applications of measurements of consumption and utilization of food by phytophagous insects. In: BHASKARAN, G.; FRIEDMAN, S.; RODRIGUEZ, J. G. (Eds.). Current topics insect endocrinology and nutrition. New York: Plenum, 1981. p. 337-362.

KOUHI, D.; NASERI, B.; GOLIZADEH, A. Nutritional performance of the tomato fruit borer, Helicoverpa armigera, on different tomato cultivars. Journal of Insect Science, Oxford, v. 14, n. 2, p. 1-12, 2014.

LIU, Z. D. et al. Effects of parental exposure to high temperature on offspring performance in the cotton bollworm, Helicoverpa armigera (Hubner) (Lepidoptera: Noctuidae): adaptive significance of the summer diapause. Applied Entomology and Zoology, Osaka, v. 39, n. 3, p. 373-379, 2004.

LIU, Z. D. et al. A true summer diapause induced by high temperatures in the cotton bollworm, Helicoverpa armigera (Lepidoptera: Noctuidae). Journal of Insect Physiology, Oxford, v. 52, n. 10, p. 1012-1020, 2006.

MARTIN, L. A.; PULIN, A. S. Host-plant specialization and habitat restriction in an endangered insect, Lycaena dispar batavus (Lepidoptera: Lycaenidae): I. Larval feeding and oviposition preferences. European Journal of Entomology, Branisovska, v. 101, n. 1, p. 51-56, 2004.

MORAL-GARCIA, F. J. Analysis of the spatiotemporal distribution of Helicoverpa armigera (Hübner) in a tomato field using a stochastic approach. Biosystems Engineering, Oxford, v. 93, n. 3, p. 253-259, 2006.

MOSCARDI, F. et al. Artrópodes que atacam as folhas da soja. In: HOFFMANN-CAMPO, B. C.; CORRÊAFERREIRA, B. S.; MOSCARDI, F. Soja: manejo integrado de insetos e outros artrópodes-praga. Brasília, DF: Embrapa, 2012. p. 213-333.

MURÚA, M. G. et al. First record of Helicoverpa armigera (Lepidoptera: Noctuidae) in Argentina. Florida Entomologist, Lutz, v. 97, n. 2, p. 854-856, 2014.

NAMIN, F. R.; NASERI, B.; RAZMJOU, J. Nutritional performance and activity of some digestive enzymes of the cotton bollworm, Helicoverpa armigera, in response to seven tested bean cultivars. Journal of Insect Science, Oxford, v. 14, n. 1, p. 1-18, 2014.

NASERI, B.; GATEhOUSE, A. M. R. Digestive proteolytic and amylolytic activities of Helicoverpa armigera in response to feeding on different soybean cultivars. Pest Management Science, Watsonville, v. 66, n. 12, p. 1316-1323, 2010.

PARAGUAY. Servicio Nacional de Calidad y Sanidad Vegetal (Senave). Paraguay: Asunción. 2013. Disponível em: <http://www.abc.com.py/edicion-impresa/economia/ senave-en-alerta-trasingreso-de-peligrosa-plagaagricola-629240.html>. Acesso em: 29 jan. 2014.

PARRA, J. R. P. Consumo e utilização de alimentos por insetos. In: PANIZZI, A. R.; PARRA, J. R. P. (Eds.). Ecologia nutricional de insetos e suas aplicações no manejo de pragas. São Paulo: Manole, 1991.

PARRA, J. R. P. Índices nutricionais para medir consumo e utilização de alimentos por insetos. In: PANIZZI, A. R.; PARRA, J. R. P. Bioecologia e nutrição de insetos: base para o manejo integrado. Brasília, DF: Embrapa Informações Tecnológicas, 2009. p. 37-90.

PARRA, J. R. P.; HADDAD, M. L. Determinação do número de instares de insetos. Piracicaba: Fealq, 1989.

POGUE, M. G. A new synonym of Helicoverpa zea (Boddie) and differentiation of adult males of $\mathrm{H}$. zea and $\mathrm{H}$. armigera (Hübner) (Lepidoptera: Noctuidae: Heliothinae). Annals of the Entomological Society of America, College Park, v. 97, n. 6, p. 1222-1226, 2004.

REED, W. Heliothis armigera (Hb.) (Noctuidae) in western Tanganyika: II. Ecology and natural and chemical control. Bulletin of Entomological Research, London, v. 56, n. 1, p. 127-140, 1965.

REED, W.; POWAR, C. S. Heliothis: a global problem. In: INTERNATIONAL WORKSHOP ON HELIOTHIS MANAGEMENT, 1982, Patancheru. Proceedings... Patancheru: Icrisat, 1982. p. 9-14.

ROGERS, D. J.; BRIER, H. B. Pest-damage relationships for Helicoverpa armigera (Hubner) (Lepidoptera: Noctuidae) on soybean (Glycine max) and dry bean (Phaseolus vulgaris) during pod-fill. Crop Protection, Brighton, v. 29, n. 1, p. 47-57, 2010. 
RUAN, Y. M.; WU, K. J. Performances of the cotton bollworm, Helicoverpa armigera, on different food plants. Acta Entomology, Bohemoslov, v. 44, n. 2, p. 205-212, 2001.

SALVADORI, J. R.; PEREIRA, P. R. V. da S.; SPECHT, A. Helicoverpa armigera no Sul. Cultivar Grandes Culturas, Pelotas, n. 176, p. 22-23, 2013.

SALVADORI, J. R.; SUZANA, C. S. Saldo da Helicoverpa. Cultivar Grandes Culturas, Pelotas, n. 187, p. 26-28, 2014.

SHARMA, H. C. et al. Detached leaf assay to screen for host plant resistance to Helicoverpa armigera. Journal of Economic Entomology, Lanham, v. 98, n. 2, p. 568-576, 2005.

SLANKY JUNIOR, F.; WHEELER, G. S. Caterpillars compensatory feeding response to diluted nutrients leads to toxic allelochemical dose. Entomologia Experimentalis et Applicata, Dordrecht, v. 65, n. 2, p. 171-186, 1987.
SPECHT, A. et al. Identificação morfológica e molecular de Helicoverpa armigera (Lepidoptera: Noctuidae) e ampliação de seu registro de ocorrência no Brasil. Pesquisa Agropecuária Brasileira, Brasília, DF, v. 48, n. 6, p. 689692, 2013.

SULLIVAN, M.; MOLET, T. CPHST pest datasheet for Helicoverpa armigera. Chicago: USDA-APHIS-PPQCPHST, 2014.

TAY, W. T. et al. A brave new world for an old world pest: Helicoverpa armigera (Lepidoptera: Noctuidae) in Brazil. Plos One, San Francisco, v. 8, n. 1, p. 134, 2013.

ZALUCKI, M. P. et al. The biology and ecology of Helicoverpa armigera (Hübner) and $H$. punctigera Wallengren (Lepidoptera: Noctuidae) in Australia: what do we know? Australian Journal of Zoology, Melbourne, v. 34, n. 6, p. 779-814, 1986. 